\title{
TOPICAL TACROLIMUS 0.1\% FOR TREATMENT OF CUTANEOUS MICROCYSTIC LYMPHATIC MALFORMATIONS
}

\author{
S.A. Salvia, M.A. Amore, C.M. Papendieck
}

Phlebology and Lymphology Unit (SAS,MAA), Cardiovascular Surgery Division, Central Military Hospital, Buenos Aires; Angiopediatria Foundation (CMP); and Lymphology Unit (MAA), University Hospital, Favaloro Foundation, Argentina

\section{ABSTRACT}

Microcystic lymphatic malformations as described in the international literature form a subgroup of low-flow congenital vascular malformations (VM) resulting from irregular embryological development. Microcystic lesions normally manifest as an accumulation of lymph- and blood-filled vesicles that, when externalized, cause skin maceration with consequent pain and potential infection resulting in the impairment of the patient's quality of life. There is no consensus on a standardized algorithm nor clear guidelines for successful treatment of this type of lymphatic malformation, and treatment options employed often result in ambivalent and transient outcomes with a high rate of recurrence. The topical formulation of tacrolimus is a well-known FDAapproved anti-T cell agent that was recently identified as a potent activator of ALK1, which is involved in several processes and functions including angiogenesis. We investigated if topical administration of tacrolimus may be an effective therapy for directly targeting cutaneous microcystic lymphatic malformations as a complement to systemic treatment. The study enrolled four patients with cutaneous microcystic lymphatic malformations: three male (ages: 13,15,18) and one female (age: 30). Two of the patients presented lesions on their backs, one patient on the left hand and one on the left lower limb. All four patients received treatment with topical tacrolimus $0.1 \%$ twice a day for 10 weeks on a previously selected area for application. Weekly clinical follow-ups were conducted along with close physician-patient contact. All patients displayed a satisfactory response after treatment. Lymphorrhea and bleeding were stopped in all cases and the esthetic aspect of lesions improved in two patients. To date, all patients presented no clinically significant changes to the size or extension of the lesion. Topical tacrolimus treatment is a promising and reasonable option for microcystic lymphatic malformations. Our results encourage further exploration in larger populations with the consideration that it is a safe and effective alternative or complementary therapy to systemic treatment.

Keywords: topical tacrolimus, lymphatic malformations, microcystic, vascular malformations

Microcystic lymphatic malformations are described in the international literature as a subgroup of low-flow congenital vascular malformations (VM) resulting from irregular embryological development. Their presentation is varied and they can present as macro or micro cysts affecting any soft tissue, viscera or even skin. Normally, microcystic lesions manifest as an accumulation of lymph- and blood-filled 
vesicles that, when externalized cause skin maceration with consequent pain, opportunistic infections, aesthetic deterioration due to abnormal scars and sometimes even anemia. The natural clinical evolution without treatment is a progressive deterioration causing a worsening quality of life for the patient $(1,2)$.

There is still no universal consensus on a standardized algorithm nor clear guidelines for the successful treatment of cystic lymphatic malformations. Options employed have ambivalent, transient, and incomplete results with a high rate of recurrence.

Recently, research has focused on the development of therapies targeting the activation of intracellular signaling pathways in consequence of specific genetic mutations. The new theranostic therapeutic proposal opens up new molecular therapies applying specific genomic expression strategies and thus bringing the laboratory to the patient's bedside. We believe that the future of this kind of treatment will be led by sequencing in order to plan its modified expression according to the individual mutation. Considering that most of these malformations share similar angiogenic downstream pathways, targeted therapies may be applied to several vascular anomalies. Mutations most often occur in genes that encode proteins that are part of the RAS/BRAF/ MEK/ERK and PI3 kinase/mTOR pathways, and most lymphatic malformations are associated with the latter $(3,4)$. A series of drugs are being used for such purposes, such as mTOR inhibitors (sirolimus, everolimus and temsirolimus), PI3K inhibitors (alpelisib, idelalisib, copanlisib and taselisib) $(5,6)$, AKT inhibitors (hydrochloride, Miransertib), BRAF inhibitor (vemurafenib), MEK inhibitors (trametinib, cobimetinib, U0126 or PD98059), ALK5 inhibitor (SB- 431542), ERK5 inhibitors (XMD8-92, XMD17-105), MEK5 inhibitor (BIXO2189), ABL inhibitor (ponatinib), and finally multiple kinase inhibitors (sorafenib). In addition, TIE2 kinase inhibitors are currently being developed $(7,8)$.

Sirolimus is an immunosuppressive agent indicated for prophylaxis of organ rejection in patients receiving renal transplants. Several studies have demonstrated the efficacy of systemic sirolimus therapy for vascular tumors associated with life-threatening coagulopathy and extratruncular vascular malformations including lymphatic and venous malformation caused by inappropriate activation of the PI3K/AKT/mTOR pathways with promising results (9-12). Topical sirolimus has been used and was found to be a safe, effective, non-invasive, and well-tolerated treatment for a variety of superficial complex vascular anomalies, decreasing their size and associated complications by reducing blebs, lymphorrhea, and bleeding (13-17).

Tacrolimus, also an immunosuppressive agent, involves the inhibition of calcinerin and ultimately decreases $\mathrm{T}$-cell activation and differentiation of CD4 T-cells. The resulting down-regulation of $\mathrm{T}$-cell activity and the inhibition of transcription of many proinflammatory cytokines results in its anti-inflammatory and immunomodulatory action. Furthermore, tacrolimus inhibits the production of $\mathrm{TNF} \alpha$, a factor involved in angiogenesis. It is primarily indicated for prophylaxis of organ rejection in patients receiving allogeneic liver, renal, or heart transplants. Its topical formulation is a well-known FDA-approved anti-Tcell agent used to treat cutaneous inflammatory/fibrotic diseases such as atopic dermatitis and other immune-mediated inflammatory dermatoses (psoriasis, contact eczema, rosacea, vitiligo, lichen planus).

In the last decade a new BMP9/ALK1/ENG/SMAD pathway has been described. ENG and ALK1 proteins are involved in several processes and functions, including angiogenesis, vasodilatation, proliferation/apoptosis, cytoskeletal organization, leukocyte extravasation and hemostasis, and the immune system, among others. Interestingly, ALK1 has also recently been reported to be readily expressed by lymphatic endothelial cells and ALK1 signaling may also play a role in lymphangiogenesis (18). Given the crucial role of endoglin/ALK1 in angiogenesis (19), various drugs, including bevacizumab, sorafenib, thalidomide, metformin, and propranolol, have been used to inhibit angiogenesis by modulating some endoglin/ALK1dependent functions. Subsequently, ALK1 is 
considered an emerging target for antiangiogenic therapy in cancer disease $(20,21)$. The loss of ALK1 function in endothelial cells leads to enhanced activity of the PI3K pathway $(22,23)$. This ALK1 loss of function is found in hereditary hemorrhagic telangiectasia (HHT), also known as Rendu-OslerWeber syndrome. Tacrolimus seems to be a reasonable new treatment option considering its anti-angiogenic properties. The consideration of this drug arose through the fortuitous case report of a patient with HHT who received a liver transplant and was treated with tacrolimus in order to prevent rejection. One month after the treatment, internal and external telangiectases, epistaxes, and anemia had disappeared $(24,25)$.

Recently, studies have shown that tacrolimus enhances the signaling and gene expression of ALK1 loss-of-function, improves the vascular pathology, and opposes the proangiogenic gene expression associated with HHT $(26,27)$. Findings on the association between the BMP9/ALK1/ENG/SMAD pathway and how PI3 kinase inhibition improves vascular malformations in mouse models in HHT have also been reported. It is important to note that both tacrolimus and sirolimus have been identified as activators of ALK1 signaling (28), but tacrolimus has been shown to be more effective for this purpose. Finally, a clinical study has been published on the efficacy of a tacrolimus $0.1 \%$ nasal ointment as a treatment for epistaxis in HHT (29).

Our aim is to attempt to determine whether topical administration may be an effective therapy. This type of administration cannot achieve circulating levels or penetrate for deeper involvement, but it can be a complement to systemic treatment.

\section{OBJECTIVE}

We aim to assess the efficacy of $0.1 \%$ topical tacrolimus in microcystic lymphatic malformation in order to avoid clinical complications such as bleeding and lymphorrhagia.

\section{METHODS}

We studied four patients with cutaneous microcystic lymphatic malformations. They comprised three males (ages: $13,15,18)$ and one female (age: 30 ). Two of the patients presented lesions on the lower back, one on the left hand, and one on the left lower limb. All received treatment with topical tacrolimus $0.1 \%$ twice a day for 10 weeks on a previously selected area for application.

Weekly clinical follow-ups were conducted along with close physician-patient contact. The primary outcomes assessed were a decrease in number of episodes of bleeding/ lymphorrhagia and reduction in size of lesions and local inflammation. Each patient received information about the risks and benefits, and accepted and signed informed consent.

\section{RESULTS}

Treatment was evaluated once a week and a progressive improvement of lesions was observed with improvement increasing over the weeks. Ten weeks after the initiation of therapy, all patients demonstrated a satisfactory response. Lymphorrhea, bleeding, and local inflammation were interrupted in all cases, and the aesthetic aspect of lesions improved in two patients. Microcystics lesions had involuted, and were found empty of content. Episodes of bleeding and lymphorrhea had ceased.

After treatment, two patients continued with oral rapamycin $1 \mathrm{mg}$ twice a day due to the type of malformation and deep tissue involvement.

To date, all patients presented no clinically significant changes regarding size or extension of lesions (Fig. 1).

\section{DISCUSSION}

Microcystic lymphatic malformations do not currently have a standardized treatment and present with a complication of frequent formation of opening of these vesicles to the outside resulting in maceration, pain, aberrant healing, and bacterial superinfection. This considerably affects patients' quality of life and demands special care and healing of the 

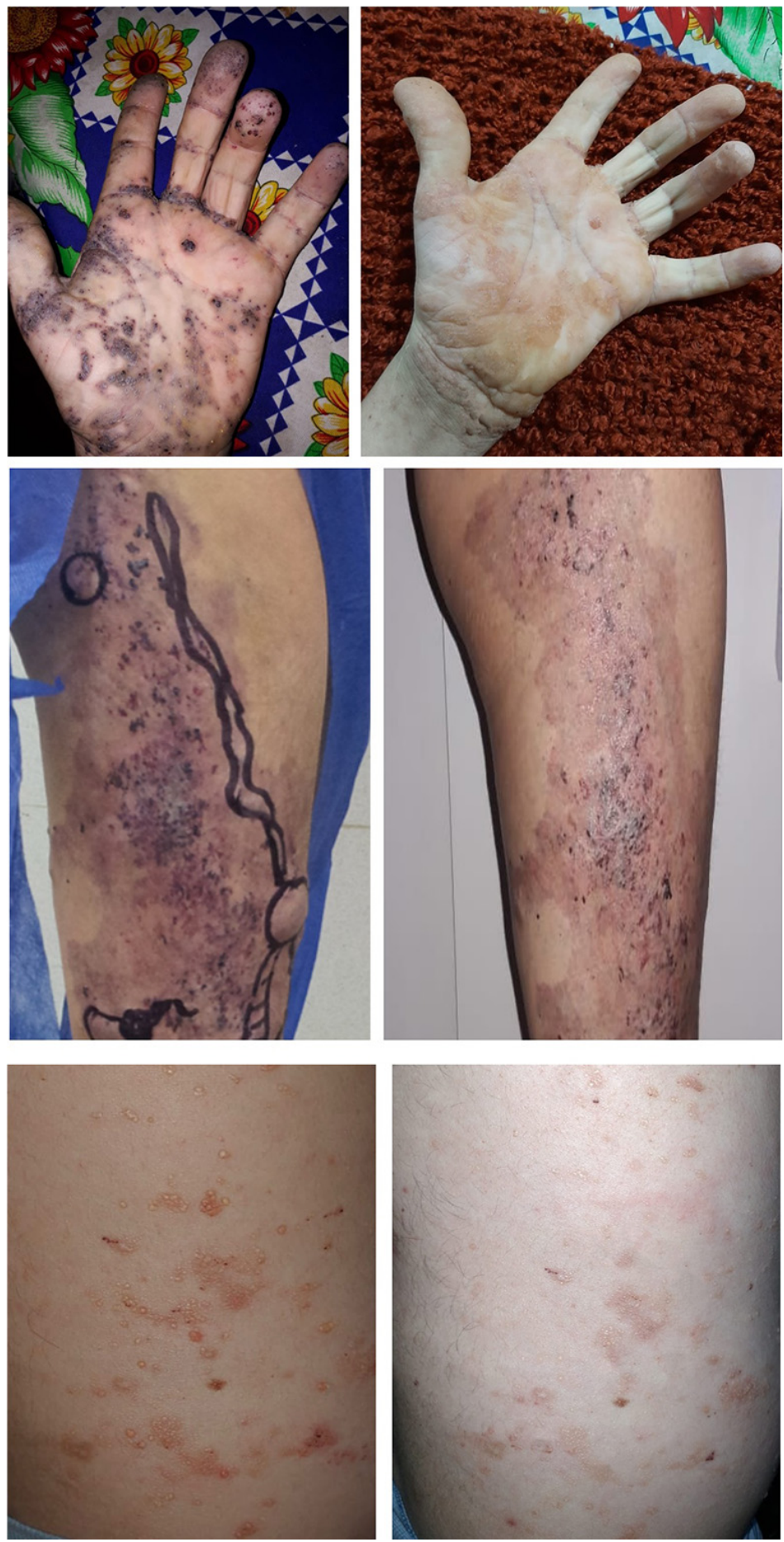

Fig 1. Images of three different patients (top, middle, and bottom) before (left) and after (right) 10 week treatment with $0.1 \%$ topical tacrolimus for microcystic lymphatic malformations. All three patients demonstrate a reduction in lesion size and fluid. 
lesions. The natural evolution of these lesions without directed treatment is a progressive worsening. The current treatment options described and employed present ambivalent, transient, and unrealistic results with a high rate of recurrence.

We are encouraged by the current developing theranostic future therapy in vascular malformations based on knowledge of the sequencing of affected genes and targeting the cascade pathways of defective genomic signaling. Given the very high costs, the possibility of a diagnosis based on accurate genomic mapping in our country is completely beyond the reach of both the physician and the patient. In order to bridge this gap and be able to offer the patient an adequate treatment, we consider that the concordant phenotypic expression among different patients would indicate a common underlying mechanism.

Although we do not ignore the fact that the cutaneous manifestation of lesions often embodies only the tip of the iceberg in the pathology, we believe that a topical therapy applied at the site of the lesion can significantly help to improve the patient's quality of life. Many of our patients present deep and systemic lesions, so topical tacrolimus was employed as an adjunct to systemic therapy. The topical formulation of tacrolimus is a well-known FDA-approved anti-T-cell agent. It has a good biosecurity profile and is used to treat a large number of cutaneous inflammatory and fibrotic diseases.

In the last decade a new BMP9/ALK1/ ENG/SMAD pathway has been described, wherein ENG and ALK1 proteins are involved in several processes and functions, including angiogenesis, vasodilatation, proliferation/ apoptosis, cytoskeletal organization, leukocyte extravasation and hemostasis and the immune system. Recently a report has identified tacrolimus and sirolimus as activators of ALK1 signaling. Using this information, we attempted to determine if topical tacrolimus administration could be an effective therapy for microcystic lymphatic lesions on a patient's skin, recognizing that this type of administration cannot achieve circulating levels or penetrate to the deeper components. Our results were very positive with all patients demonstrating an involution of microvesicles, which were emptied of their contents, and a cessation of episodes of bleeding and lymphatic leakage, thus improving their quality of life significantly. To date, none of them have presented significant changes or suffered new episodes or complications.

\section{CONCLUSION}

Topical Tacrolimus treatment is a promising and reasonable option for the treatment of microcystic lymphatic lesions. We encourage its use and consider it to be a safe and effective adjuvant therapy. Further studies with larger number of patients are needed to confirm its use for these microcystic lymphatic malformations.

\section{CONFLICT OF INTEREST AND DISCLOSURE}

The authors declare no competing financial interests exist.

\section{REFERENCES}

1. International Society for the Study of Vascular Anomalies. Classification of vascular anomalies. 2018. Available from: https://issva. org/classification. Accessed 31 July, 2021.

2. Greene, AK, CA Perlyn, AI Alomari: Management of lymphatic malformations. Clin. Plast. Surg. 38 (2011), 75-82.

3. Kim, JS, SK Hwang, Chung, HY: The molecular pathophysiology of vascular anomalies: Genomic research. Arch. Plast. Surg. 47 (2020), 203-208.

4. Adams, DM: Practical genetic and biologic therapeutic considerations in vascular anomalies. Tech. Vasc. Interventional Rad. 22 (2019), 100629

5. di Blasio, L, A Puliafito, PA Gagliardi, et al: $\mathrm{PI} 3 \mathrm{~K} / \mathrm{mTOR}$ inhibition promotes the regression of experimental vascular malformations driven by PIK3CA-activating mutations. Cell Death Dis. 9 (2018), 45.

6. Hanker, AB, V Kaklamani, CL Arteaga: Challenges for the clinical development of PI3K inhibitors: Strategies to improve their impact in solid tumors. Cancer Discov. 9 (2019), 482-491. 
7. Zúñiga-Castillo, M, CL Teng, JMC Teng: Genetics of vascular malformation and therapeutic implications. Curr. Opin. Pediatr. 3 (2019), 498-508.

8. Van Damme, A, E Seront, V Dekeuleneer, et al: New and emerging targeted therapies for vascular malformations. Am. J. Clin. Dermatol. 21 (2020) , 657-668.

9. Mizuno, T, T Fukuda, C Emoto, et al: Developmental pharmacokinetics of sirolimus: implications for precision dosing in neonates and infants with complicated vascular anomalies. Pediatr. Blood Cancer 64 (2017), doi: 10.1002/pbc.26470.

10. Ozeki, M, A Nozawa, $S$ Yasue, et al: The impact of sirolimus therapy on lesion size, clinical symptoms, and quality of life of patients with lymphatic anomalies. Orphanet J. Rare Dis. 14 (2019), 141.

11. Freixo, C, V Ferreira, J Martins, et al: Efficacy and safety of sirolimus in the treatment of vascular anomalies: A systematic review. J. Vasc. Surg. 71 (2020), 318-327.

12. Lee, B-B: Sirolimus in the treatment of vascular anomalies. J. Vasc. Surg. 71 (2020), 328

13. Garcia-Montero, P, J Del Box, E BaselgaTorres, et al: Use of topical rapamycin in the treatment of lymphatic malformations. J. Am. Acad. Dermatol. 80 (2019), 508-515.

14. Cai, R, H Gu, Z Yu, et al: Topical rapamycin in superficial lymphatic malformation: Microcystic lymphatic malformation or verrucous venous malformation? J. Am. Acad. Dermatol. pii: S0190-9622 (2019), 30165-3. Online ahead of print.

15. Triana, P, M Dore, VN Cerezo, et al: Sirolimus in the treatment of vascular anomalies. Eur. J. Pediatr. Surg. 27 (2017), 86-90.

16. Dodds, M, M Tollefson, L Castelo-Soccio, et al: Treatment of superficial vascular anomalies with topical sirolimus: A multicenter case series. Pediatr. Dermatol. 37 (2020), 1-6.

17. Le Sage, S, M David, J Dubois, et al: Efficacy and absorption of topical sirolimus for the treatment of vascular anomalies in children: A case series. Pediatr. Dermatol. 35 (2018), 472-477.

18. Niessen, K, G Zhang, JB Ridgway, H Chen, $M$ Yan: ALK1 signaling regulates early postnatal lymphatic vessel development. Blood 115 (2010), 1654-1661.

19. Roman, BL, AP Hinck: ALK1 signaling in development and disease: New paradigms. Cell Mol. Life Sci. 74 (2017), 4539-4560.

20. Venkatesha, VA, A Joshi, M Venkataraman, et al: P7170, a novel inhibitor of mTORC1/ mTORC2 and Activin receptor-like Kinase 1 (ALK1) inhibits the growth of non small cell lung cancer. Mol. Cancer 13 (2014), 259.

21. Cunha, SI, K Pietras: ALK1 as an emerging target for antiangiogenic therapy of cancer. Blood 117 (2011), 6999-7006.

22. Alsina-Sanchís, E, Y García-Ibáñez, AM Figueiredo, et al: ALK1 loss results in vascular hyperplasia in mice and humans through PI3K activation. Arterioscler. Thromb. Vasc. Biol. 38 (2018), 1216-1229.

23. Bischoff, J: PTEN connection in HHT2. Arterioscler. Thromb. Vasc. Biol.38 (2018), 984-985.

24. Ruiz-Llorente, L, E Gallardo-Vara, E Rossi, et al: Endoglin and ALK1 as therapeutic targets for hereditary hemorrhagic telangiectasia, Expert Opin. Ther. Targets 21 (2017), 933-947.

25. Dupuis-Girod, S, AL Chesnais, I Ginon, et al: Long-term outcome of patients with hereditary hemorrhagic telangiectasia and severe hepatic involvement after orthotopic liver transplantation: A single-center study. Liver Transplant. 16 (2010), 340-347.

26. Ruiz, S, P Chandakkar, H Zhao, et al: Tacrolimus rescues the signaling and gene expression signature of endothelial ALK1 loss-offunction and improves HHT vascular pathology. Hum. Molec. Gen. 26 (2017), 4786-4798.

27. Albiñana, V, F Sanz-Rodríguez, L RecioPoveda, et al: Immunosuppressor FK506 increases endoglin and activin receptor-like kinase 1 expression and modulates transforming growth factor- $\beta 1$ signaling in endothelial cells. Mol. Pharmacol. 79 (2011), 833-843.

28. Robert, F, A Desroches-Castan, S Bailly, et al: Future treatments for hereditary hemorrhagic telangiectasia. 1. Orphanet J. Rare Dis. 15 (2020) , 4.

29. Dupuis-Girod, S, AE Fargeton, V Grobost, et al: Efficacy and safety of a $0.1 \%$ tacrolimus nasal ointment as a treatment for epistaxis in hereditary hemorrhagic telangiectasia: A double-blind, randomized, placebocontrolled, multicenter trial. J. Clin. Med. 9 (2020), 1262.

\author{
Miguel A. Amore, MD \\ Cachimayo 2842 do piso. \\ CABA. Caballito \\ Buenos Aires, ARGENTINA CP 1424 \\ Telephone \#: 54-11-5-0466768 \\ e-mail: miguelangelamore@hotmail.com
}

